\title{
Cardiovascular Dysfunction with Low Cardiac Output Due to a Small Heart in Patients with Chronic Fatigue Syndrome
}

\author{
Kunihisa Miwa $^{1}$ and Masatoshi Fujita ${ }^{2}$
}

\begin{abstract}
Objective Little attention has been paid to possible cardiovascular involvement in patients with chronic fatigue syndrome (CFS), although many of their symptoms and signs suggest cardiovascular dysfunction. Possible cardiovascular symptoms and cardiac function were investigated in CFS patients.

Methods Cardiovascular symptoms were intensively investigated and cardiac function was evaluated echocardiographically.

Patients Fifty-three patients ( 23 men and 30 women, mean age: $31 \pm 7$ years) with CFS under 50 years were studied.

Results Slender build (body mass index $<20 \mathrm{~kg} / \mathrm{m}^{2}$ ) was common (47\%). Possible cardiovascular symptoms including shortness of breath (32\%), dyspnea on effort (28\%), rapid heartbeat (38\%), chest pain (43\%), fainting (43\%), orthostatic dizziness $(45 \%)$ and coldness of feet $(42 \%)$, were all frequent complaints. Hypotension $(28 \%)$ was occasionally noted. Electrocardiograms frequently revealed right axis deviation $(21 \%)$ and severe sinus arrhythmia (34\%) suggesting accentuated parasympathetic nervous activity. Small heart shadow (cardiothoracic ratio $\leq 42 \%$ ) was noted on the chest roentgenogram in 32 patients $(60 \%)$. Echocardiographic examination demonstrated low cardiac indexes $\left(<2 \mathrm{~L} / \mathrm{min} / \mathrm{m}^{2}\right)$ with low stroke volume indexes $\left(<30 \mathrm{~mL} / \mathrm{m}^{2}\right)$ due to a small left ventricular chamber in $19(36 \%, \mathrm{p}<0.05$ vs. $8 \%$ in 36 controls). None had reduced left ventricular ejection fraction.

Conclusion Cardiovascular symptoms are common in CFS patients. Cardiac dysfunction with low cardiac output due to small left ventricular chamber may contribute to the development of chronic fatigue as a constitutional factor in a considerable number of CFS patients.
\end{abstract}

Key words: chronic fatigue syndrome, low cardiac output, small heart, cardiothoracic ratio, cardiac function

(Inter Med 48: 1849-1854, 2009)

(DOI: 10.2169/internalmedicine.48.2347)

\section{Introduction}

The chronic fatigue syndrome affects many young people in today's modern, stressful society; it is characterized by persistent or relapsing, severe disabling fatigue, not resolved by rest, causing a marked reduction of working activity (15). Although the fatigue is often accompanied by multiple unexplained symptoms including headache, sore throat, selfreported impairment in concentration and short-term mem- ory, sleep disturbances, and musculoskeletal pain (1-5), the etiology of chronic fatigue syndrome remains unclear. Various factors have been implicated in this peculiar syndrome $(1,3-6)$. However, unreasonably the symptoms and signs have rarely been related to cardiovascular impairment or dysfunction.

Small heart syndrome has previously been reported by Master (7) as so-called "neurocirculatory asthenia" in young adults associated with a small heart shadow on the chest roentgenogram. The most frequent complaints are fatigue or

${ }^{1}$ Department of Internal Medicine, Nanto Family and Community Medical Center, Nanto, Toyama and ${ }^{2}$ Human Health Sciences, Kyoto University Graduate School of Medicine, Kyoto

Received for publication April 8, 2009; Accepted for publication July 13, 2009

Correspondence to Dr. Kunihisa Miwa, miwa.kunihisa@city.nanto.lg.jp 
weakness, rapid heart, chest pain, shortness of breath, nervousness, trembling, sweating and fainting. These symptoms are also common in patients with chronic fatigue syndrome. Recently, we reported that a small heart shadow on the chest roentgenogram is common in patients with chronic fatigue syndrome (8). Echocardiographic evaluation demonstrated that cardiac function was actually impaired with low cardiac output due to a small heart in many of the patients with chronic fatigue syndrome. In addition, the cardiac function fluctuated during the exacerbation and remission phases directly related to the severity of their symptoms (9).

In the present study, possible cardiovascular symptoms were intensively investigated and cardiac function was evaluated echocardiographically to clarify how prevalent cardiovascular impairment or dysfunction is in young adults with chronic fatigue syndrome.

\section{Methods}

\section{Study population}

After alternative medical and psychiatric causes of chronic fatigue-inducing illness had been excluded, chronic fatigue syndrome was diagnosed according to the revised case definition of Fukuda et al (10) as follows. Chronic fatigue syndrome was defined by the presence of the following: 1) self-reported, unexplained, persistent or relapsing fatigue lasting six or more consecutive months; is not substantially alleviated by rest; and results in marked reduction in previous levels of occupational, educational, social, or personal activities; and 2) the concurrent occurrence of four or more of the following eight symptoms: self-reported impairment in short-term memory or concentration severe enough to cause substantial reduction in previous levels of occupational, educational, social, or personal activities; sore throat; tender cervical or axillary lymph nodes; muscle pain; multijoint pain without joint swelling or redness; headaches of a new type, pattern, or severity; unrefreshing sleep; and postexertional malaise lasting more than 24 hours.

The study population comprised 53 Japanese patients (23 men and 30 women: mean age $31 \pm 7$ years) with chronic fatigue syndrome who were younger than 50 years old. None of them had muscle atrophy detectable by the physical examination.

\section{Symptoms and physical examination}

All of the study patients underwent a thorough history taking at the first visit to our institute or when the diagnosis of chronic fatigue syndrome was established. The presence or absence of cardiovascular complaints including shortness of breath, dyspnea on effort, dyspnea at rest, rapid heartbeat, chest pain, orthostatic dizziness, coldness of feet, fainting and weakness was determined. They also underwent an intensive physical examination. In particular, abnormalities in heart sounds, such as murmurs and extrasounds, coldness of feet and presence of pretibial pitting edema and epigastric splash sound were monitored. Bimanual palpability of the right kidney was also determined. The body mass index was calculated as body weight $(\mathrm{kg}) /$ body height $(\mathrm{m})^{2}$.

\section{Chest roentgenograms}

Cardiothoracic ratio (CTR) was calculated as the transverse diameter of the cardiac shadow divided by the transverse diameter of the thoracic cage at the level of diaphragm in the postero-anterior projection on the chest roentgenogram. In the present study, a CTR $\leq 42 \%$ was defined as a small heart $(8,10,11)$. Also, the presence or absence of narrow chest findings including flat chest and straight back was determined. Narrow chest was diagnosed when the anteroposterior diameter of the thoracic cage at the level of the 8th thoracic vertebra estimated in the right-to-left projection of the lateral view on the chest roentgenogram was less than $40 \%$ of the transverse diameter of the thoracic cage estimated in the postero-anterior projection.

\section{Electrocardiographic examination}

All of the study patients underwent a 3-min electrocardiographic recording following at least a 10 -min rest in the morning. RR50 (\%) was calculated as the number of differences $>50 \mathrm{~ms}$ between two successive R-R intervals divided by total heartbeats.

\section{Echocardiographic examination}

All individuals underwent standard M-mode and twodimensional echocardiography. The left ventricular (LV) dimensions were measured according to the recommendations of the American Society of Echocardiography (12). LV volume was calculated by the Teichholz' formula (13), and an ejection fraction was determined by the conventional method. Mitral valve prolapse was diagnosed according to the criteria of Gilbert et al (14) with Yoshikawa's modification (15). Echocardiographic parameters were compared between the patients and 36 age- and sex-matched healthy control subjects (15 males and 21 females with a mean age of $33 \pm 8$ years).

All studied patients gave informed consent, and the study protocol was approved by the ethics committee of our institution.

\section{Statistical analysis}

Values are presented as the mean \pm standard deviation. Comparisons of echocardiographic parameters between the study patients and the controls were performed with Student unpaired t-test. Proportional data were analyzed by the chisquare test, with Yates' correction. The value of significance was set at $\mathrm{p}<0.05$.

\section{Results}

The results are summarized in Tables 1, 2 and also in Fig. 1. As shown in Table 1, minor criteria symptoms by the revised case definition (10) were often found. In addition, 
Table 1. Clinical Profiles and Cardiovascular Complaints and Symptoms in 53 Study Chronic Fatigue Syndrome Patients

Minor criteria symptoms

$\begin{array}{ll}\text { Difficulty concentrating } & 41(77 \%) \\ \text { Forgetfulness } & 36(68 \%) \\ \text { Sore throat } & 22(42 \%) \\ \text { Neck or axillary tenderness } & 17(32 \%) \\ \text { Myalgia } & 26(49 \%) \\ \text { Arthralgia } & 16(30 \%) \\ \text { Headache } & 32(62 \%) \\ \text { Sleep disturbance or unrefreshing sleep } & 39(74 \%) \\ \text { Postexertional malaise } & 38(72 \%)\end{array}$

Possible cardiovascular symptoms

Shortness of breath

Dyspnea on effort

Rapid heartbeat

Chest pain

Orthostatic dizziness

Coldness of feet

$22(42 \%)$

Fainting

$23(43 \%)$

Weakness

Other symptoms

Anorexia
Abdominal pain
Nausea
Diarrhea

possible cardiovascular symptoms including shortness of breath $(32 \%)$, dyspnea on effort $(28 \%)$, rapid heartbeat $(38 \%)$, chest pain $(43 \%)$, orthostatic dizziness $(45 \%)$, coldness of feet (42\%), fainting (43\%) and weakness $(47 \%)$ were found to be common in the study chronic fatigue syndrome patients. Also, gastrointestinal symptoms including anorexia (36\%), abdominal pain $(30 \%)$, nausea $(40 \%)$ and diarrhea $(28 \%)$ were frequent.

As shown in Table 2, hypotension was occasionally found (28\%). Pretibial pitting edema (25\%), cold feet (40\%), epigastric splash sound $(19 \%)$ and right kidney palpability $(37 \%)$ were frequently noted on physical examination. Lymph node enlargement was relatively rare $(23 \%)$. Mitral valve prolapse was suggested in some of the patients by auscultatory findings including late systolic murmur (21\%) and midsystolic click (7\%). Slender built (body mass index $<20 \mathrm{~kg} / \mathrm{m}^{2}$ ) was frequently $(47 \%)$ noted, whereas obesity (body mass index $>25 \mathrm{~kg} / \mathrm{m}^{2}$ ) was rare $(13 \%)$. Chest roentgenographic examination revealed that small heart was prevalent $(60 \%)$ in the study patients, as was narrow chest (43\%). Figure 1 shows the chest roentgenogram of a typical case with chronic fatigue syndrome and small heart.

Both right axis deviation including vertical axis (electrical axis $\geq 90^{\circ}$ ) $(21 \%$ ), and severe sinus arrhythmia (RR50 > $20 \%$ of total beats during the 3-min electrocardiogram) $(34 \%)$ were frequently noted in the electrocardiographic examination. Echocardiographic examination revealed that the mean values of LV end-diastolic and end-systolic dimensions, stroke volume indexes and cardiac indexes were all rather lower in the study CFS patients than in the control subjects, although the difference was not statistically significant. However, small values of $\mathrm{LV}$ end-diastolic dimensions ( $<41 \mathrm{~mm}: 34 \%, \mathrm{p}<0.05$ vs. $11 \%$ in controls), stroke volume indexes $\left(<30 \mathrm{~mL} / \mathrm{m}^{2}: 36 \%, \mathrm{p}<0.05\right.$ vs. $8 \%$ in controls $)$ and cardiac indexes $\left(<2 \mathrm{~L} / \mathrm{ml} / \mathrm{m}^{2}: 36 \%, \mathrm{p}<0.05\right.$ vs. $8 \%$ in controls) were significantly more prevalent in the study patients as compared with those in the controls (Table 2). Mitral valve prolapse was noted in $8(15 \%)$ of the study patients. No patient had a reduced $(<60 \%)$ left ventricular ejection fraction.

\section{Discussion}

It has been known that patients with chronic fatigue syndrome have various unexplained symptoms including headache, sore throat, self-reported impairments in concentration and short-term memory, sleep disturbances, and musculoskeletal pain. Several symptoms and physical findings are known to be prevalent among chronic fatigue syndrome patients, although none of them are specific for the diagnosis of the syndrome $(10,16)$. The subjects in the present study also had a variety of possible cardiovascular complaints, including chest pain, palpitation, dyspnea or shortness of breath, coldness of feet, dizziness and fainting, although all of these symptoms are not necessarily attributable to cardiovascular dysfunction. Frequently noted physical examination findings such as epigastric splash sound, right kidney palpability, cold feet and pretibial pitting edema, may be related to visceral ptosis with slender build, and peripheral circulatory impairment. Weakness, rapid heartbeat and orthostatic dizziness may be related to hypotension and orthostatic dysregulation. Auscultatory findings suggested typical mitral valve prolapse in some of the patients. In addition, chest roentgenographic, electrocardiographic and, echocardiographic examinations revealed several distinct findings in the present young adult patients. Specifically, a small heart shadow was often observed on the chest roentgenogram in these patients. Both small LV chamber size and poor cardiac function with low stroke volume and cardiac indexes were 
Table 2. Physical, Chest Roentgenographic, Electrocardiographic and Echocardiographic Findings of 53 Study Chronic Fatigue Syndrome Patients

\begin{tabular}{|c|c|c|c|c|}
\hline \multicolumn{5}{|l|}{ Physical findings } \\
\hline \multicolumn{2}{|l|}{ Body temperature $\left({ }^{\circ} \mathrm{C}\right)$} & \multicolumn{3}{|l|}{$36.4 \pm 0.3$} \\
\hline \multicolumn{2}{|l|}{ Heart rate (beats/min) } & \multicolumn{3}{|l|}{$73 \pm 13$} \\
\hline \multicolumn{2}{|c|}{ Blood pressure (systolic/diastolic) } & \multicolumn{3}{|c|}{$112 \pm 12 / 75 \pm 7$} \\
\hline \multicolumn{2}{|c|}{ Hypotension (Systolic blood pressure < 100mmHg) } & \multicolumn{3}{|l|}{$15(28 \%)$} \\
\hline \multicolumn{2}{|l|}{ Lymphnode swelling } & \multicolumn{3}{|l|}{$12(23 \%)$} \\
\hline \multicolumn{2}{|l|}{ Late systolic murmur } & \multicolumn{3}{|l|}{$11(21 \%)$} \\
\hline \multicolumn{2}{|l|}{ Midsystolic click } & \multicolumn{3}{|l|}{$4(7 \%)$} \\
\hline \multicolumn{2}{|l|}{ Cold feet } & \multicolumn{3}{|l|}{$21(40 \%)$} \\
\hline \multicolumn{2}{|l|}{ Pretibial pitting edema } & \multicolumn{3}{|l|}{$13(25 \%)$} \\
\hline \multicolumn{2}{|l|}{ Epigastric splash sound } & \multicolumn{3}{|l|}{$10(19 \%)$} \\
\hline \multicolumn{2}{|l|}{ Right kidney palpability } & \multicolumn{3}{|l|}{$20(37 \%)$} \\
\hline \multicolumn{2}{|c|}{ Obesity (body mass index $>25$ ) } & \multicolumn{3}{|l|}{$7(13 \%)$} \\
\hline \multicolumn{2}{|c|}{ Slender built (body mass index $<20$ ) } & \multicolumn{3}{|l|}{$25(47 \%)$} \\
\hline \multicolumn{5}{|l|}{ Chest roentgenographic findings } \\
\hline Cardiothoracic ratio $\leq 42$ & & $32(60 \%)$ & & \\
\hline Narrow chest & & $23(43 \%)$ & & \\
\hline Electrocardiographic findings & & & & \\
\hline Heart rate (beats/min) & $>100$ & $1(2 \%)$ & & \\
\hline & $<60$ & $9(17 \%)$ & & \\
\hline Right axis deviation (axi & $\left.90^{\circ}\right)$ & $11(21 \%)$ & & \\
\hline Clockwise rotation & & $9(17 \%)$ & & \\
\hline Severe sinus arrhythmia & R50>20\%) & $18(34 \%)$ & & \\
\hline Supraventricular arrhyth & & $4(8 \%)$ & & \\
\hline Ventricular arrhythmia & & $2(4 \%)$ & & \\
\hline Echocardiographic findings & & & & Controls \\
\hline Left ventricular end-dias & ic dimension (mm) & $43 \pm 6$ & NS & $45 \pm 4$ \\
\hline & $<41$ & $18(34 \%)$ & $\mathrm{p}<0.05$ & $4(11 \%)$ \\
\hline Left ventricular end-syst & c dimension (mm) & $26 \pm 5$ & NS & $27 \pm 1$ \\
\hline & $<26$ & $19(36 \%)$ & NS & $6(17 \%)$ \\
\hline Stroke volume index (m & $\left.n^{2}\right)$ & $35 \pm 8$ & NS & $39 \pm 7$ \\
\hline & $<30$ & $19(36 \%)$ & $\mathrm{p}<0.05$ & $3(8 \%)$ \\
\hline Cardiac index $(\mathrm{L} / \mathrm{min} / \mathrm{m}$ & & $2.5 \pm 0.6$ & NS & $2.8 \pm 0.6$ \\
\hline & $<2$ & $19(36 \%)$ & $\mathrm{p}<0.05$ & $3(8 \%)$ \\
\hline Ejection fraction (\%) & & $69 \pm 6$ & NS & $70 \pm 6$ \\
\hline & $<60$ & $0(0 \%)$ & NS & $0(0 \%)$ \\
\hline Mitral valve prolapse & & $8(15 \%)$ & NS & $1(3 \%)$ \\
\hline
\end{tabular}

documented echocardiographically. Reduced LV ejection fraction was not observed in any of the patients. Electrocardiograms showed severe sinus arrhythmia and vertical or right axis deviation in a considerable number of the patients, suggesting parasympathetic predominance and vertical heart position.

Recently, we have reported that "small heart" is commonly noted in chronic fatigue syndrome patients, suggesting that small heart syndrome in association with a small LV cavity and poor LV function may be related to the genesis in these patients as a predisposing factor to fatigue (8). Moreover, repeated echocardiographic examinations also revealed that hemodynamic abnormalities including smaller LV chamber size, and diminished stroke volume and cardiac output during the exacerbation phase significantly improved during the remission phase, suggesting a direct relation between the severity of chronic fatigue and impaired cardiac function in chronic fatigue syndrome patients with a small heart (9).

In 1944, Master (7) reported several young adult cases with both "small heart shadow" and "neurocirculatory asthenia" and hypothesized that the symptoms might be caused by diminished venous return, diminished cardiac output, anoxemic heart muscle and decreased oxygen saturation of the blood as a result of constitutionally small heart. Similarly, DaCosta (17) described "irritable heart" in 1871, a peculiar form of functional disorder of the heart found in the military population during the American Civil War. The disorder frequently presented after an episode of diarrhea. Fatigue was an almost universal complaint in DaCosta's syndrome, although symptoms included palpitation, cardiac pain, headache and dimness $(17,18)$. The present study showed evidence of a small heart chamber and impaired cardiac function in patients with both chronic fatigue syndrome and small heart. Diarrhea, sweating and loss of appetite may lead to dehydration and reduce venous return or preload, re- 


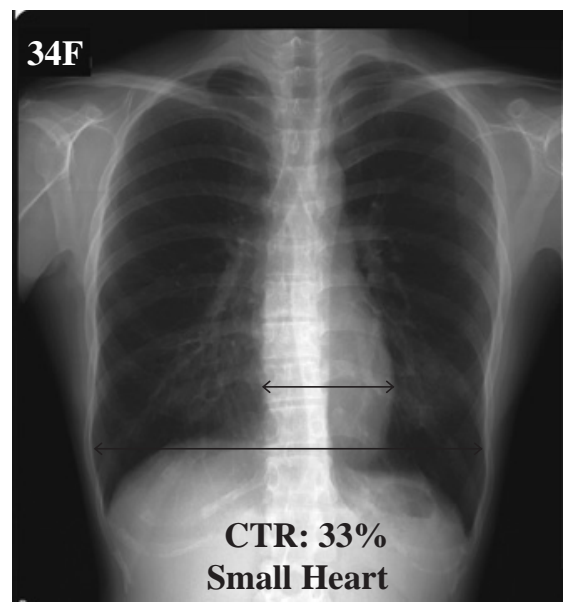

Figure 1. The chest roentgenogram of a 34-year-old female chronic fatigue syndrome patient with a "small heart" [cardiothoracic ratio (CTR): 33\%]. She had suffered from relapsing severe general malaise and easy fatigability for several years. She also frequently developed orthostatic dizziness. She frequently became unable to work as a housewife due to severe malaise and headache. The symptoms were exacerbated every summer. The upper arrow line indicates the cardiac transverse diameter, and the lower line, the thoracic transverse diameter.

sulting in further decreases in both stroke volume and cardiac output, thereby exacerbating symptoms in young chronic fatigue syndrome patients with small heart. Komaroff et al (16), based on analysis of the experiences from one large center, reported that gastrointestinal symptoms including anorexia and nausea, discriminated chronic fatigue syndrome patients from the control and the patients with other conditions. Preload reduction due to dehydration may contribute to the development and exacerbation of symptoms via further lowering cardiac output in many of these patients.

Patients with small heart are known to have slender physiques with low body mass indexes, frequently visceral ptosis with wandering kidney, asthenia, nervousness as well as foot coldness, suggesting physical, autonomic nervous and psychological irritability or lack of relaxation $(6,9,17)$. Autonomic nervous dysfunction with accentuated basal parasympathetic tone may be associated with these symptoms through inhibiting compensatory sympathetic activation which otherwise contributes to preserve cardiac function.

Although muscle atrophy was not evident in the study patients, it is possible that total muscle volume has been reduced due to immobility in the patients as compared with the controls. It cannot be excluded that low cardiac output is attributable in part to the decreased demand by the loss of muscle volume. Further investigation is needed to clarify the causal relationship between the low cardiac output observed in the patients and the symptoms of chronic fatigue syndrome.

In conclusion, possible cardiovascular symptoms including dyspnea on effort, palpitation, chest pain, fainting, orthostatic dizziness and coldness of feet, are often complaints of young adult chronic fatigue syndrome patients. Electrocardiograms frequently showed severe sinus arrhythmia, suggesting exaggerated parasympathetic nervous function. Echocardiographic examination demonstrated poor LV function resulting from a small LV chamber in a considerable number of these patients. Thus, impaired cardiac function with low output appears to contribute to the development of chronic fatigue as a constitutional factor in a considerable number of patients with chronic fatigue syndrome.

\section{References}

1. Shafran SD. The chronic fatigue syndrome. Am J Med 90: 730739, 1991.

2. Holmes GP, Kaplan JE, Gantz NM, et al. Chronic fatigue syndrome: A working case definition. Ann Int Med 108: 387-389, 1988.

3. Afari N, Buchwald D. Chronic fatigue syndrome: A review. Am J Psychiatry 160: 221-236, 2003.

4. Reeves WC, Wagnor D, Nisenbaum R, et al. Chronic fatigue syndrome-A clinically empirical approach to its definition and study. BMC Medicine 3: 19-27, 2005.

5. Klonoff DC. Chronic fatigue syndrome. Clin Inf Dis 15: 812-823, 1992.

6. Miwa K, Fujita M. Increased oxidative stress by low serum vitamin $\mathrm{E}$ concentrations in patients with chronic fatigue syndrome. Int J Cardiol 136: 238-239, 2009.

7. Master AM. Neurocirculatory asthenia due to small heart. Med Clin North Am 28: 577-588, 1944.

8. Miwa K, Fujita M. "Small heart syndrome" in patients with chronic fatigue syndrome. Clin Cardiol 31: 328-333, 2008.

9. Miwa K, Fujita M. Cardiac function fluctuates during exacerbation and remission in young adults with chronic fatigue syndrome and "small heart". J Cardiol 54: 29-35, 2009.
10. Fukuda K, Straus SE, Hickle I, et al. The chronic fatigue syndrome: A comprehensive approach to its definition and study. Ann Intern Med 121: 953-959, 1994.

11. Abe T. The small heart syndrome. Asian Med J 33: 295-302, 1990.

12. Schiller NB, Shah PM, Crawford M, et al. Recommendations for the quantification of the left ventricle by two-dimensional echocardiography. J Am Soc Echocardiogr 2: 358-367, 1989.

13. Teichholz LE, Kreulen T, Herman MV, Gorlin R. Problems in echocardiographic volume determinations: Echocardiographicangiographic correlations in the presence or absence of asynergy. Am J Cardiol 37: 7-11, 1976.

14. Gilbert BW, Schatz RA, Van Ramm OT, Behar VS, Kisslo JA. Mitral valve prolapse: Two-dimensional echocardiographic and angiographic correlation. Circulation 54: 716-723, 1976.

15. Yoshikawa J, Kato H, Yanagihara K, et al. Criteria for the diagnosis of prolapsed mitral valve using phonocardiography and echocardiography. J Cardiography 12: 773-777, 1982 (in Japanese, Abstract in English).

16. Komaroff AL, Fagioli LR, Geiger AM, et al. An examination of the working case definition of chronic fatigue syndrome. Am J Med 100: 56-64, 1996. 
Inter Med 48: 1849-1854, 2009 DOI: 10.2169/internalmedicine.48.2347

17. DaCosta JM. An irritable heart; a clinical study of a form of functional cardiac disorder and its consequences. Am J Med Sci 121: $17-53,1871$.

18. Wooley CF. Where are the diseases of yesteryear? DaCosta's syn- drome, soldiers heart, the effort syndrome, neurocirculatory asthenia-And the mitral valve prolapse syndrome. Circulation 53: 749-751, 1976

(C) 2009 The Japanese Society of Internal Medicine http://www.naika.or.jp/imindex.html 